\title{
Quality of life after bariatric surgery - a comparative study of laparoscopic banding vs. bypass
}

\author{
Müller, M K ; Wenger, C ; Schiesser, M ; Clavien, P A ; Weber, M
}

\begin{abstract}
Laparoscopic gastric banding and Roux-en-Y gastric bypass are widely used for the treatment of morbid obesity. The impact of these two procedures on health-related quality of life has not been analyzed in comparative studies. METHODS: A matched-pair analysis of a prospectively collected database was performed. Fifty-two consecutive patients with laparoscopic gastric bypass were randomly matched to fifty-two patients with laparoscopic gastric banding according to age, BMI, and gender. Quality of life was assessed using two standardized questionnaires (SF 36 and Moorehead-Ardelt II). RESULTS: Mean preoperative BMI was $45.7 \mathrm{~kg} / \mathrm{m}(2)$ for the bypass patients and $45.3 \mathrm{~kg} / \mathrm{m}(2)$ for the banding patients. Mean BMI after 3 years follow-up of was $30.4 \mathrm{~kg} / \mathrm{m}(2)$ and $33.1 \mathrm{~kg} / \mathrm{m}(2)(\mathrm{p}=0.036)$. In the SF 36 questionnaire, gastric bypass patients yielded a mean total score of 613 versus 607 points in the gastric banding group ( $\mathrm{p}=0.543$ ), which is comparable to the normal population in Europe. In the MoorheadArdelt II questionnaire, the gastric bypass patients scored a mean total of 1.35 points and the gastric banding patients 1.28 points $(\mathrm{p}=0.747)$. Of the patients, $97 \%$ with a gastric bypass and $83 \%$ with a gastric banding were satisfied with the result of the operation $(\mathrm{p}=0.145)$. CONCLUSION: The patients after laparoscopic gastric bypass and laparoscopic gastric banding have a high level of satisfaction 3 years after the operation and have similar quality of life scores compared to the normal population. Quality of life indexes were not different between the two procedures and were independent of weight loss in successfully operated patients.
\end{abstract}

DOI: https://doi.org/10.1007/s11695-008-9522-y

Posted at the Zurich Open Repository and Archive, University of Zurich

ZORA URL: https://doi.org/10.5167/uzh-8312

Journal Article

Published Version

Originally published at:

Müller, M K; Wenger, C; Schiesser, M; Clavien, P A; Weber, M (2008). Quality of life after bariatric surgery - a comparative study of laparoscopic banding vs. bypass. Obesity Surgery, 18(12):1551-1557.

DOI: https://doi.org/10.1007/s11695-008-9522-y 


\title{
Quality of Life After Bariatric Surgery-A Comparative Study of Laparoscopic Banding vs. Bypass
}

\author{
Markus K. Müller • Christa Wenger • Marc Schiesser • \\ Pierre-Alain Clavien • Markus Weber
}

Received: 15 February 2008 / Accepted: 27 March 2008/Published online: 7 May 2008

(C) Springer Science + Business Media, LLC 2008

\begin{abstract}
Laparoscopic gastric banding and Roux-en-Y gastric bypass are widely used for the treatment of morbid obesity. The impact of these two procedures on healthrelated quality of life has not been analyzed in comparative studies.

Methods A matched-pair analysis of a prospectively collected database was performed. Fifty-two consecutive patients with laparoscopic gastric bypass were randomly matched to fifty-two patients with laparoscopic gastric banding according to age, BMI, and gender. Quality of life was assessed using two standardized questionnaires (SF 36 and Moorehead-Ardelt II).

Results Mean preoperative BMI was $45.7 \mathrm{~kg} / \mathrm{m}^{2}$ for the bypass patients and $45.3 \mathrm{~kg} / \mathrm{m}^{2}$ for the banding patients. Mean BMI after 3 years follow-up of was $30.4 \mathrm{~kg} / \mathrm{m}^{2}$ and $33.1 \mathrm{~kg} / \mathrm{m}^{2}(p=0.036)$. In the SF 36 questionnaire, gastric bypass patients yielded a mean total score of 613 versus 607 points in the gastric banding group $(p=0.543)$, which
\end{abstract}

\footnotetext{
Authors' contributions 1. Markus K. Müller: Lead investigator, study design, data collection, statistical analysis, preparation of the manuscript. 2. Christa Wenger: Co-investigator, data collection, statistical analysis, preparation of the manuscript. 3. Marc Schiesser: Statistical analysis, data interpretation, preparation of the manuscript. 4. Pierre-Alain Clavien: Data interpretation, preparation and final approval of the manuscript. 5. Markus Weber: Data interpretation, preparation and final approval of the manuscript.
}

Remarks: The Moorehead-Ardelt Quality of life Questionnaire II is copyright protected and requires a licensing agreement to be used.

M. K. Müller · C. Wenger · M. Schiesser · P.-A. Clavien •

M. Weber $(\triangle)$

Division of Visceral and Transplantation Surgery,

University Hospital Zürich, Rämistrasse 100,

8091 Zurich, Switzerland

e-mail: markus.weber@usz.ch is comparable to the normal population in Europe. In the Moorhead-Ardelt II questionnaire, the gastric bypass patients scored a mean total of 1.35 points and the gastric banding patients 1.28 points $(p=0.747)$. Of the patients, $97 \%$ with a gastric bypass and $83 \%$ with a gastric banding were satisfied with the result of the operation $(p=0.145)$. Conclusion The patients after laparoscopic gastric bypass and laparoscopic gastric banding have a high level of satisfaction 3 years after the operation and have similar quality of life scores compared to the normal population. Quality of life indexes were not different between the two procedures and were independent of weight loss in successfully operated patients.

Keywords Quality of life · Bariatric surgery .

Laparoscopic gastric banding $\cdot$ Laparoscopic gastric bypass

\section{Introduction}

Obesity surgery is currently one of the most frequently performed surgical procedures in the US and Europe with steadily increasing numbers [1]. For 2006 more than 200,000 operations were estimated [2]. Over the past few years, many surgeons have learned to perform this type of surgery laparoscopically rather than using an open approach [3], and currently the laparoscopic gastric banding and the laparoscopic Roux-en-Y gastric bypass are the most widely used bariatric procedures [4].

Only very few centers have a large experience with both laparoscopic procedures and therefore attempts to compare the two procedures have suffered from methodological flaws with inadequate demographic data $[5,6]$. The discussion is currently limited to the claim from the advocates of 
either procedure that their choice is valid because of their "good results". Both procedures are associated with advantages and disadvantages. The laparoscopic gastric banding represents the lesser invasive bariatric procedure compared to bypass and has the potential of full reversibility [7]. On the other hand, bypass procedures are associated with a larger weight loss and positively affect comorbidities more efficiently [8]. To date, comparative studies are mainly looking at clinical data of weight loss, change of comorbidities and morbidity [9], but only few comparative studies on health-related quality of life exist [10,11], and not all investigated procedures were done laparoscopically.

Obesity is associated with many comorbidities, significantly impairs quality of life and patients are exposed to discriminating prejudices $[12,13]$. Several studies suggest that quality of life is improved after bariatric procedures like gastric bypass $[14,15]$ as well as after gastric banding $[16,17]$, but it has not been investigated if these procedures differently affect quality of life.

The ideal study design to compare two procedures would be a prospective controlled randomized trial. Unfortunately, such study is likewise not feasible due to the important difference in the invasiveness and irreversibility of these procedures. The next best strategy to compare these procedures is a matched-pair study design using a large prospectively collected database. Laparoscopic gastric banding and laparoscopic gastric bypass have become routine procedures at our institution since 1995, and we have established a large prospective database including all patients undergoing bariatric procedures. Using this database, we performed a matchedpair analysis to evaluate the impact of both approaches on quality of life.

\section{Patients and Methods}

Over 500 laparoscopic banding procedures and more than 600 laparoscopic proximal bypass operations were performed by three bariatric consultant surgeons at our institution since May 1995. Data for each patient were prospectively collected in our bariatric database.

Patients, operated between October 1997 and October 2004, were randomly matched according to gender, age, and preoperative body mass index. We were able to match 142 patients, 71 pairs, with their primary bariatric procedure in place. Of these, 17 were lost to follow-up, and finally, 125 were contacted. The questionnaires were sent to the patients via mail, and after 2 and 4 weeks, the patients were contacted via telephone in case of missing answers. Thirteen did not answer the questionnaires. We received answers from 112 patients. The rate of return was consequently $90 \%$. When a pair was incomplete, we rematched the redundant patients according to the matching criteria. Finally, we were able to match 52 pairs of patients treated either by laparoscopic gastric banding or laparoscopic gastric bypass. The median time between operation and follow-up of all patients was 3.1 (range: 1.5-7.9) years, banding patients were assessed after a median time of 5.9 (range: 1.5-7.9) years postoperatively, whereas bypass patients were assessed after a median time of 2.5 (1.54.3) years after the operation.

We choose one general quality of life questionnaire, the SF 36, and the obesity-specific Moorehead-Ardelt quality of life questionnaire II (MA II). Additionally, we developed a center-specific questionnaire.

The indication for obesity surgery was the same in both groups according to federal regulations [18]. Inclusion criteria and indication for bariatric surgery were as follows: BMI $>40 \mathrm{~kg} / \mathrm{m}^{2}$ or BMI $>35 \mathrm{~kg} / \mathrm{m}^{2}$ with comorbidities, history of obesity more than 5 years, failed conservative treatment for more than 2 years, and age between 18 and 60 years. The choice for the type of surgery was based on the time when the operation was performed. In the first period of the study (from October 1997 until June 2000), we preferentially performed laparoscopic banding procedures as bariatric operation. Based on the growing evidence from the USA demonstrating the feasibility of laparoscopic gastric bypass, we progressively switched to this procedure after June 2000.

\section{Operative Technique}

The laparoscopic gastric banding, using a $10 \mathrm{~cm}$ Lap Band System (Bioenterics ${ }^{\mathrm{TM}}$, Santa Barbara, CA, USA), was performed as published before [19]. The band was placed in a perigastric position creating a pouch of $15-25 \mathrm{ml}$ size. The device was fixed with four to six gastro-gastric stitches. The reservoir was not filled until the fourth postoperative week.

The bypass procedure was performed like described by Wittgrove and Clark [20]. The stomach was transected creating a pouch of $25 \mathrm{ml}$ size. The jejunum was transected $50 \mathrm{~cm}$ distal to the doudeno-jejunal flexure. A stapled side to side jejuno-jejunostomy was created with a Roux limb length of $150 \mathrm{~cm}$. The Roux limb was positioned antecolic and the gastro-jejunostomy was performed using a circular stapler (CEA $25 \mathrm{~mm}$, Tyco $^{\circledR}$, Mansfield, MA, USA).

In the postoperative course, all the patients received a contrast study of the esophagus and stomach after 1 day in case of a banding and after 3 days in case of a bypass procedure. Resumption of oral diet was started in absence of a leakage and if a prompt passage was documented by gastrographin follow-through. The patients were discharged as soon as sufficient oral fluid and soft food intake was possible. 
Study Endpoints

\section{Patient Characteristics}

Preoperative assessment collected all patient characteristics and medical data.

\section{Weight Change}

Postoperative assessment was performed after 1, 3, 6 and 12 months, thereafter yearly. Weight change was reported as excess weight loss and according to the latest recommendations in excess BMI lost [21].

\section{Quality of Life Questionnaires}

We used the SF 36 questionnaire and the MooreheadArdelt quality of life questionnaire II (MA II). Additionally a third center specific questionnaire, developed at our institution, was used.

The SF 36 questions addresses the two main topics of physical and mental wellbeing [22]. It is divided into the following eight aspects: physical functioning, role-physical, bodily pain, general health, vitality, mental health, roleemotional and social functioning. Norm-based scores for each aspect range from 0 to 100 with a mean of 50 and a standard deviation of 10 (US population).

The MA II questionnaire has six dimensions. The MA II questionnaire is used as a part of the Bariatric Analysis and Reporting Outcome System (BAROS) and assesses the patients subjective impression of quality of life in the areas of general self-esteem, physical activity, social contacts, satisfaction concerning work, pleasure related to sexuality and focus on eating behavior. Each item is scored from -0.5 to +0.5 . The total score consists of the sum of the six dimensions with a range of -3 to +3 . A total score of 1.1 to 2 is considered "good", above 2.1 is "very good" [23].

The center-specific questionnaire assessed patients marital status, sports activities, satisfaction with given infor-

Table 1 Patients characteristics

\begin{tabular}{|c|c|c|c|}
\hline & Bypass & Banding & $p$ value \\
\hline$n$ & 52 & 52 & \\
\hline Age & $40.1(10.8)$ & $40.7(10.1)$ & 0.253 \\
\hline Gender (female/male) & $45 / 7$ & $45 / 7$ & 1.000 \\
\hline ASA & $2.21(0.7)$ & $2.19(0.8)$ & 0.829 \\
\hline BMI kg/m² & $45.7(4.28)$ & $45.3(4.4)$ & 0.740 \\
\hline Preoperative weight $\mathrm{kg}$ & $124(14.2)$ & $122(14.1)$ & 0.522 \\
\hline
\end{tabular}

Values are means (standard deviation)
Table 2 Clinical outcome at time of quality of life evaluation

\begin{tabular}{lccc}
\hline & Bypass & Banding & $p$ value \\
\hline BMI kg/m & $30.4(3.6)$ & $33.1(6.8)$ & 0.036 \\
Excess BMI lost \% & $75.1(14.9)$ & $58.1(33.2)$ & 0.007 \\
EWL \% & $62.9(13.8)$ & $47.9(26.6)$ & 0.001 \\
Reoperations & 5 & 6 & 1.000 \\
\hline
\end{tabular}

Values are means (standard deviation); Reoperations were four internal herniations and one incisional hernia in the bypass group and six rebanding procedures in the banding group.

mation, overall satisfaction, and if patients would undergo this operation again.

Statistical Analysis

Patient weight and outcome data were prospectively collected. Analysis was performed using standard software (SPSS 12.0 for Windows). To compare continuous variables between the two groups the Mann-Whitney $U$ test was used. Categorical variables were compared using the chisquare test or when appropriate, Fischer's exact test was applied. To compute predicting factors for quality of life, uni- and multivariate regression analysis was performed for the SF 36 as well as for the MA II questionnaires. Results are expressed as means and standard deviation (SD), unless otherwise stated. A $p$ value of less than 0.05 was considered to indicate statistical significance.

\section{Results}

Were Both Patients Groups Comparable?

As a consequence of the matching process, the two groups were comparable regarding age, gender, and preoperative BMI. The study enrolled 45 women and 7 men corresponding pairs. Mean age was 40.1 years for the gastric bypass patients and 40.7 years for the gastric banding patients. Mean preoperative BMI was $45.7 \mathrm{~kg} / \mathrm{m}^{2}$ for the bypass patients and $45.3 \mathrm{~kg} / \mathrm{m}^{2}$ for the banding patients. Mean ASA score was comparable between the two groups, $p=0.829$ (Table 1).

What was the Clinical Outcome of the Two Procedures?

Mean BMI at the time of quality of life evaluation was $30.4 \mathrm{~kg} / \mathrm{m}^{2}$ in the bypass group and $33.1 \mathrm{~kg} / \mathrm{m}^{2}$ for the banding patients $(p=0.036)$. This corresponds to an excess weight loss of $62.9 \%$ for the bypass and $47.9 \%$ for the banding group ( $p=0.001)$, as well as an excess BMI loss of $75 \%$ and $58 \%(p=0.007)$, respectively (Table 2 ). 
Table 3 SF 36 quality of life questionnaire

\begin{tabular}{lccc}
\hline & Bypass & Banding & $p$ value \\
\hline Physical function & 84.2 & 80.2 & \\
Role physical & 84.0 & 87.4 & \\
Bodily pain & 71.7 & 60.9 & \\
General health & 74.3 & 71.3 & \\
Vitality & 66.2 & 62.1 & \\
Mental health & 71.8 & 77.3 & \\
Role emotional & 88.0 & 90.4 & \\
Social functioning & 72.7 & 77.6 & \multirow{2}{*}{0.543} \\
Total score & 612.8 & 607.2 & \\
\hline
\end{tabular}

The SF 36 assesses the two main topics of physical and mental wellbeing. It is divided into the following eight aspects: physical functioning, role-physical, bodily pain, general health, vitality, mental health, role-emotional and social functioning. Norm-based scores range form 0 to 100 with a mean of 50 and a standard deviation of 10

Five patients in the bypass group (10\%) and six patients in the banding group (11\%) had to undergo a reoperation during the follow-up period. Reoperations were four internal herniations and one incisional hernia in the bypass group and six rebanding procedures for slippage in the gastric banding group.

Diabetes was present in nine of the gastric bypass patients and in seven of the gastric banding patients preoperatively. Diabetes was resolved in $100 \%$ in the bypass group and four (57\%) in the banding group. In three gastric banding patients $(43 \%)$, diabetes was still present.

\section{Is There a Difference in Quality of Life After Bypass} or Banding?

There was no significant difference in quality of life between the two groups using SF 36 and MA II quality of life questionnaires.

For the SF 36, gastric bypass patients yielded a total score of 613 versus 607 points in the gastric banding group

Table 4 Moorehead-Ardelt quality of life questionnaire II (MA II)

\begin{tabular}{llll}
\hline & Bypass & Banding & $p$ value \\
\hline General self-esteem & 0.25 & 0.26 & \\
Physical activity & 0.24 & 0.18 & \\
Social contacts & 0.29 & 0.30 & \\
Satisfaction concerning work & 0.30 & 0.24 & \\
Pleasure related to sexuality & 0.12 & 0.19 & \\
Focus on eating behavior & 0.16 & 0.12 & \\
Total score & 1.35 & 1.28 & 0.747 \\
\hline
\end{tabular}

The Moorehead-Ardelt quality of life questionnaire II: Each item is scored from -0.5 to +0.5 . The total score consists of the sum of the six dimensions with a range of -3 to +3 . A total score below -2.1 is very poor, -2 to -1.1 is poor, -1 to +1 is fair 1.1 to 2 is good, above 2.1 is very good.
Table 5 Univariat analysis of factors influencing quality of life (SF 36 questionnaire)

\begin{tabular}{lrrrr}
\hline & \multicolumn{1}{c}{$B$} & \multicolumn{2}{c}{$95 \%$ Confidence interval } & $p$ value \\
\hline Bypass operation & -6.95 & -68.37 & 54.47 & 0.822 \\
Age & -3.60 & -6.47 & -0.74 & 0.014 \\
Gender female & -22.58 & -117.31 & 72.14 & 0.637 \\
Married & 70.08 & 10.13 & 130.04 & 0.023 \\
Sports activity & 51.65 & -17.76 & 121.06 & 0.142 \\
Actual BMI & -2.43 & -8.57 & 3.71 & 0.433 \\
EWL & 0.25 & -1.30 & 1.79 & 0.753
\end{tabular}

In the multivariate analysis only the marital status (married) was an independent variable for improved quality of life in the total SF 36 score

( $p=0.543$; Table 3 ). These results correspond to the results of a normal European population [24]. For the physical health score, the gastric bypass group yielded 314 points and the gastric banding patients 300 points. Furthermore, for the mental health score, gastric bypass patients got 299 points and gastric banding patients 307 points.

In the Moorhead-Ardelt questionnaire, the gastric bypass patients scored a total of 1.35 points and the gastric banding patients 1.28 points ( $p=0.747$; Table 4 ). Twentysix patients with banding and 21 patients with bypass achieved between 1.1 and 2 points, which corresponds to a "good" score and 9 patients with banding and 14 patients with bypass scored more than 2.1 points, which corresponds to a "very good" score.

Which Factors Influence Quality of Life?

We performed a univariate and multivariate regression analysis to define factors influencing quality of life in all 104 patients. Age and marital status were found to be statistically significant in a univariat regression analysis for the SF 36. In the univariat analysis of the Moorhead-Ardelt

Table 6 Univariat analysis of factors influencing quality of life (MA II questionnaire)

\begin{tabular}{lrrcc}
\hline & \multicolumn{1}{c}{ B } & \multicolumn{2}{c}{$95 \%$ Confidence interval } & $p$ value \\
\hline Bypass operation & 0.07 & -0.39 & 0.53 & 0.758 \\
Age & -0.01 & -0.04 & 0.01 & 0.248 \\
Gender female & 0.86 & 0.18 & 1.55 & 0.014 \\
Married & 0.70 & 0.26 & 1.14 & 0.002 \\
Sports activity & 0.90 & 0.39 & 1.42 & 0.001 \\
Actual BMI & -0.05 & -0.10 & -0.01 & 0.019 \\
EWL & 0.01 & -0.01 & 0.02 & 0.127 \\
\hline
\end{tabular}

In the multivariate analysis only female gender, marital status, and sport activity were independent variables for improved quality of life in the total MA II score. 
total score, these were female gender, actual BMI, marital status, and physical activity.

In the multivariate analysis of the SF 36 total score, only the marital status (married) was the only independent factor for better quality of life scores (B: 92.9; CI: 26.4-159.5; $p=0.007)$. For the Moorhead-Ardelt II total score, the marital status (B: 0.7; CI: 0.2-1.1; $p=0.004)$, female gender (B: 0.9 ; CI: $0.1-1.7 ; p=0.023$ ) and sports activity (B: 0.9; CI: $0.3-1.4 ; p=0.002$ ) were independent variables for improved quality of life (Tables 5 and 6).

Of note, at the time of quality of life evaluation, 21 $(40 \%)$ patients in the bypass group and $26(50 \%)$ patients in the banding group were married.

Were the Expectations Met and Would the Patients Do it Again?

With a center-specific questionnaire, the patients were asked how satisfied they were with the result of the operation. Of the patients, 97\% with a gastric bypass and $83 \%$ with a gastric banding stated to be satisfied with the operation ( $p=0.145$; Fig. 1 ). Of the patients, $89 \%$ in the bypass group and $95 \%$ in the banding group $(p=0.419)$ would undergo the operation again. Almost all the patients reported to have been very well informed about the operation: $100 \%$ of the gastric bypass patients and $97 \%$ in the banding group $(p=0.474)$.

$73 \%$ of the bypass patients and $68 \%$ of the banding patients reported to perform regular sports $(p=0.793)$. Furthermore, $79 \%$ of the bypass patients and $60 \%$ of the banding patients claimed to do even more sports than before the operation $(p=0.088)$. In addition, the patients were asked, whether they were happy with their actual body: 31 (74\%) of the bypass and $23(64 \%)$ of the banding patients are happy with their body. Of the patients, $73 \%$ of

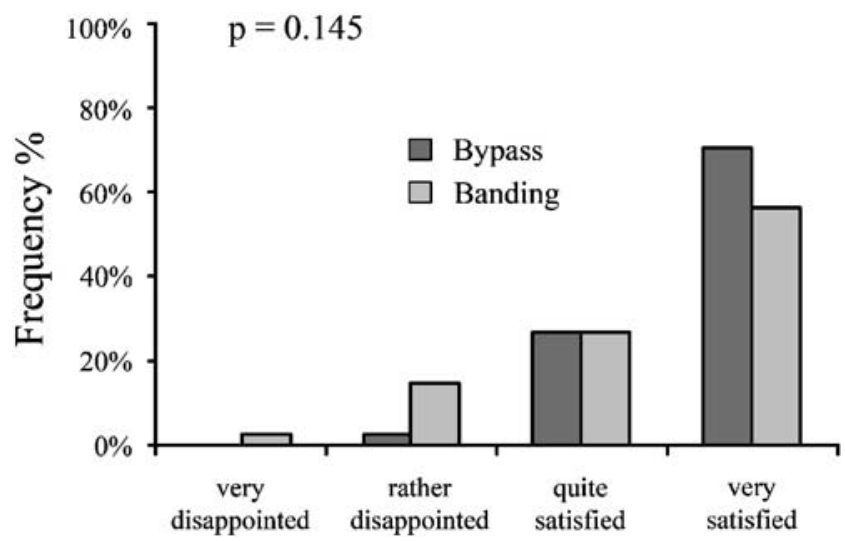

Fig. 1 Satisfaction with the result of the operation. Gastric bypass patients were very satisfied in $70 \%$ and quite satisfied in $27 \%$, patients with banding were very satisfied in $56 \%$ and quite satisfied in $27 \%$ the bypass group and $78 \%$ of the banding group $(p=0.606)$ still wanted to lose more weight.

\section{Discussion}

In this single center study, we found that patients after gastric bypass and gastric banding achieve good quality of life scores. In comparison of the two procedures, bypass patients lose more weight, and comorbidities are treated more effectively. Quality of life seems equally affected with no difference between the two procedures. Factors influencing quality of life were female gender, marital status, and sports activities, but not the type of operation or the actual BMI, nor the excess weight lost. Overall, 97\% of the bypass patients and $83 \%$ of the banding patients were satisfied with the result of the operation, and nine in ten patients would undergo their respective operation again.

Several comparative studies suggest that bypass affects the clinical aspects of weight loss and comorbidities more positively than the banding procedure [9, 25, 26]. Quality of life is improved by bariatric procedures in general [27]. Quality of life on the other hand has only been investigated sparsely in comparison of the two most frequent performed bariatric procedures, laparoscopic gastric banding and laparoscopic gastric bypass [10]. These procedures have a different impact on the eating abilities of the patients. The banding procedure is more restrictive, whereas patients after bypass are occasionally affected by a dumping syndrome. Thus, it has been hypothesized, that quality of life is different after these two procedures.

The quality of a study comparing two different surgical therapies relies on the similarity of the patient demographics between the groups and the constancy of a surgical team at a high volume center [28]. To meet these requirements, we choose a matched-pair methodology in the setting of a single center study in an institution with more than 100 bariatric procedures per year. By matching, for age, sex, and BMI, we established a homogeneous patient demographic between the groups with minimal selection bias. The treatment by one single team involving only three surgeons additionally provided an equal standard of care throughout the study. Quality of life in this study focuses on health-related quality of life and excludes aspects of life not directly related to health, like income or freedom. To date, many quality of life questionnaires are available with different reliability and validity. As an obesity-specific questionnaire, we choose the Moorehead-Ardelt quality of life II questionnaire because it is easy to perform and interpret, and it has been used in many bariatric studies before [29]. In the medical literature, the SF 36 questionnaire is widely used and well validated in all types of studies as well as in the obesity literature [27]. 
It is noteworthy that we could achieve a high return rate when mailing out the questionnaires to the patients and calling them by phone in case of a missing answer. This high acceptance of the original Moorehead-Ardelt quality of life questionnaire by the patients was already stated in a previous paper and highlights the utility of this questionnaire [11]. The guidelines for Reporting of the American Society for Bariatric Surgery claim a minimum of $60 \%$ follow up to reach statistical significance which was achieved in this study [30].

In the SF 36, our patients achieved in the follow-up, with scores between 62 and 90 per item, comparable scores to a standard population in Europe [24]. In the MoreheadArdelt II questionnaire, the mean total score was 1.28 and 1.35 , which is a "good" result according to the developers definition [23]. This indicates that operated patients can achieve an almost normal quality of life score after bariatric surgery, whereas non-operated obese patients seem to have severe psychological problems in the long term [31].

Other studies have demonstrated a positive effect of bariatric surgery on quality of life in a longitudinal study design, assessing preoperative and follow-up data. The improvement of quality of life was long lasting as shown in the SOS study with 10 years follow-up. In this study, improvement of quality of life was highly related to weight loss. It was suggested that already a maintained weight loss of only $10 \%$ was sufficient for a positive long-term effect on quality of life [32].

This study was not designed to compare pre- and postoperative quality of life scores in a longitudinal manner, but to compare two different operative procedures. There was no measurable difference between the two procedures regarding quality of life. This might be explained by the fact that the possible differences of the two procedures are not reflected by the questionnaires. Even though the weight loss was different between the groups, the weight loss in both groups was higher than the suggested threshold of $10 \%$ in the SOS study. Thus, we hypothesize that all our patients improved the quality of life, and the difference between the two procedures was below detection limit. This indicates that patients after bariatric surgery lose enough weight, even if not down to ideal weight, so the overall satisfaction is good, as shown in our specific questions. Even if a majority wants to lose more weight, patients are satisfied and would undergo the operation again.

Some possible limitations have to be considered when interpreting these data. First, this is a selected group of banding patients because the patients with band failure (insufficient weight loss) or severe esophageal dysmotility were converted to a bypass procedure and consequently were not assessed with a questionnaire. The aim of the study was to investigate patients who were successfully operated with one single procedure (laparoscopic banding vs. bypass) and not patients with two consecutive ones. In our initial experience with banding patients, a high percentage developed esophageal dysmotility disorders or did not lose weight sufficiently. As a consequence, $30 \%$ of all patients operated with banding during the study period had the band removed and were converted to a bypass procedure. Consequently, this study concludes only on patients who were successfully operated with one procedure. Nevertheless, as the indications have changed for banding in the last years (this group was one of the first to report on failures of laparoscopic bandings and conversion to bypass procedures [33]), it is acknowledged that well-selected patients benefit from bandings and successfully lose weight $[16,34]$. These patients are good candidates for laparoscopic gastric banding and would still have the procedure done today. And even though there were reoperations in the remaining banding group as well as in the bypass group, patients with reoperations still achieve good quality of life scores.

Second, the time between the operation and the quality of life questionnaires is not the same in both groups. Nevertheless, it is long enough so patients are in wellestablished and a stable phase, which is usually after 18 to 24 months, as has been shown in long-term studies [26], and a comparison can be made.

In summary, this study demonstrates that patients after laparoscopic gastric bypass and laparoscopic gastric banding have a "good" quality of life, as measured by the MA II. This finding was reinforced by the SF 36 that also demonstrated postoperative quality of life scoring similar to normal values. Quality of life indexes were not different between the two operation procedures, as long as the banding procedure was successful without conversion to another procedure in the long term.

Acknowledgment This study was supported by a research grant from Covidien Switzerland Ltd.

Competing interest statements Nothing to disclose.

\section{References}

1. Nguyen NT, Root J, Zainabadi K, et al. Accelerated growth of bariatric surgery with the introduction of minimally invasive surgery. Arch Surg. 2005;140(12):1198-202, discussion 1203.

2. Shapiro S. Bariatric Surgery Complication Rates High In Some Hospitals, New HealthGrades Ratings And Study Show. www. medicalnewstoday.com 2007.

3. Schirmer B. Laparoscopic bariatric surgery. Surg Endosc. 2006;20 Suppl 2:S450-5.

4. Nguyen NT, Silver M, Robinson M, et al. Result of a national audit of bariatric surgery performed at academic centers: a 2004 University HealthSystem Consortium Benchmarking Project. Arch Surg. 2006;141(5):445-9; discussion 449-50.

5. DeMaria EJ, Sugerman HJ, Kellum JM, et al. Results of 281 consecutive total laparoscopic Roux-en-Y gastric bypasses to treat morbid obesity. Ann Surg. 2002;235(5):640-5; discussion 645-7. 
6. Biertho L, Steffen R, Ricklin T, et al. Laparoscopic gastric bypass versus laparoscopic adjustable gastric banding: a comparative study of 1,200 cases. J Am Coll Surg. 2003;197(4):536-44; discussion 544-5.

7. Belachew M, Zimmermann JM. Evolution of a paradigm for laparoscopic adjustable gastric banding. Am J Surg. 2002; 184(6B):21S-5S

8. Pories WJ, Swanson MS, MacDonald KG, et al. Who would have thought it? An operation proves to be the most effective therapy for adult-onset diabetes mellitus. Ann Surg. 1995;222(3):339-50; discussion 350-2.

9. Weber M, Muller MK, Bucher T, et al. Laparoscopic gastric bypass is superior to laparoscopic gastric banding for treatment of morbid obesity. Ann Surg. 2004;240(6):975-82; discussion 982-3.

10. Hell E, Miller KA, Moorehead MK, Norman S. Evaluation of health status and quality of life after bariatric surgery: comparison of standard Roux-en-Y gastric bypass, vertical banded gastroplasty and laparoscopic adjustable silicone gastric banding. Obes Surg. 2000;10(3):214-9.

11. Wolf AM, Falcone AR, Kortner B, Kuhlmann HW. BAROS: an effective system to evaluate the results of patients after bariatric surgery. Obes Surg. 2000;10(5):445-50.

12. Lang T, Hauser R, Schlumpf R, et al. [Psychological comorbidity and quality of life of patients with morbid obesity and requesting gastric banding]. Schweiz Med Wochenschr. 2000;130(20): 739-48.

13. Flanagan SA. Obesity: The Last Bastion of Prejudice. Obes Surg. 1996;6(5):430-7.

14. Dymek MP, le Grange D, Neven K, Alverdy J. Quality of life and psychosocial adjustment in patients after Roux-en-Y gastric bypass: a brief report. Obes Surg. 2001;11(1):32-9.

15. de Zwaan M, Lancaster KL, Mitchell JE, et al. Health-related quality of life in morbidly obese patients: effect of gastric bypass surgery. Obes Surg. 2002;12(6):773-80.

16. O'Brien PE, Dixon JB, Brown W, et al. The laparoscopic adjustable gastric band (Lap-Band): a prospective study of mediumterm effects on weight, health and quality of life. Obes Surg. 2002; 12(5):652-60.

17. Horchner R, Tuinebreijer W. Improvement of physical functioning of morbidly obese patients who have undergone a Lap-Band operation: one-year study. Obes Surg. 1999;9(4):399-402.

18. Bundesgesetz über die Krankenversicherung der Schweizerischen Eidgenossenschaft. Anhang 1 des Artikels 1 der Leistungsverordnung (in Kraft ab 1.1.2000).

19. Belachew M, Legrand MJ, Defechereux TH, et al. Laparoscopic adjustable silicone gastric banding in the treatment of morbid obesity. A preliminary report. Surg Endosc. 1994;8(11):1354-6.
20. Wittgrove AC, Clark GW, Tremblay LJ. Laparoscopic gastric bypass, Roux-en-Y: preliminary report of five cases. Obes Surg. 1994;4(4):353-7.

21. Deitel M, Gawdat K, Melissas J. Reporting weight loss 2007. Obes Surg. 2007;17(5):565-8.

22. McHorney CA, Ware JE Jr, Raczek AE. The MOS 36-Item ShortForm Health Survey (SF-36): II. Psychometric and clinical tests of validity in measuring physical and mental health constructs. Med Care. 1993;31(3):247-63.

23. Moorehead MK, Ardelt-Gattinger E, Lechner H, Oria HE. The validation of the Moorehead-Ardelt Quality of Life Questionnaire II. Obes Surg. 2003;13(5):684-92.

24. Ellert U, Bellach BM. [The SF-36 in the Federal Health Surveydescription of a current normal sample]. Gesundheitswesen. 1999;61 Spec No:S184-90.

25. Mognol P, Chosidow D, Marmuse JP. Laparoscopic gastric bypass versus laparoscopic adjustable gastric banding in the super-obese: a comparative study of 290 patients. Obes Surg. 2005;15(1):76-81.

26. Sjostrom L, Lindroos AK, Peltonen M, et al. Lifestyle, diabetes, and cardiovascular risk factors 10 years after bariatric surgery. N Engl J Med. 2004;351(26):2683-93.

27. Ballantyne GH. Measuring outcomes following bariatric surgery: weight loss parameters, improvement in co-morbid conditions, change in quality of life and patient satisfaction. Obes Surg. 2003; 13(6):954-64.

28. Courcoulas A, Perry Y, Buenaventura P, Luketich J. Comparing the outcomes after laparoscopic versus open gastric bypass: a matched paired analysis. Obes Surg. 2003;13(3):341-6.

29. Duval K, Marceau P, Perusse L, Lacasse Y. An overview of obesity-specific quality of life questionnaires. Obes Rev. 2006; 7(4):347-60.

30. Deitel M. From the Editor's Desk. Obes Surg. 2000;10(1):1

31. Buddeberg-Fischer B, Klaghofer R, Krug L, et al. Physical and psychosocial outcome in morbidly obese patients with and without bariatric surgery: a 4 1/2-year follow-up. Obes Surg. 2006;16(3):321-30.

32. Karlsson J, Taft C, Ryden A, et al. Ten-year trends in healthrelated quality of life after surgical and conventional treatment for severe obesity: the SOS intervention study. Int J Obes (Lond). 2007;31:1248-61.

33. Weber M, Muller MK, Michel JM, et al. Laparoscopic Roux-en-Y gastric bypass, but not rebanding, should be proposed as rescue procedure for patients with failed laparoscopic gastric banding. Ann Surg. 2003;238(6):827-33; discussion 833-4.

34. Dixon JB, Dixon ME, O'Brien PE. Pre-operative predictors of weight loss at 1-year after Lap-Band surgery. Obes Surg. 2001; 11(2):200-7. 